\title{
C2 Body Fracture: Report of Cases Managed Conservatively by Philadelphia Collar
}

\author{
Rouzbeh Motiei-Langroudi ${ }^{1}$, Homa Sadeghian ${ }^{2}$ \\ ${ }^{1}$ Division of Neurosurgery, Department of Surgery, Pastor Hospital, Bam University of Medical Sciences, Bam, Iran \\ ${ }^{2}$ Neurovascular Research Laboratory, Department of Radiology, Massachusetts General Hospital, Harvard Medical School, Charlestown, MA, USA
}

Study Design: Case series.

Purpose: To present results of conservative management in patients with pure C2 body fractures.

Overview of Literature: Axis body fractures, a less common subgroup of C2 fractures, are commonly classified as vertical coronal, vertical sagittal, and transverse subtypes. While the treatment paradigm for other $\mathrm{C} 2$ fractures is clear, there is insufficient evidence to support treatment guidelines for $\mathrm{C} 2$ body fractures.

Methods: Eleven patients with pure C2 body fractures were managed with external immobilization and followed thereafter.

Results: All neurologic examinations were normal. In computed tomography (CT) scans, four, two, three, and two patients had a coronal, sagittal, horizontal, and burst fracture, respectively. Magnetic resonance imaging showed hematoma and partial rupture in the anterior longitudinal ligament in four patients, posterior ligamentous complex injury in one, and normal ligamentous structure in six. All fractures were managed conservatively using the Philadelphia collar, which was continued until complete disappearance of symptoms (within 1-3 months in all patients). The decision to discontinue the neck collar was made by a dynamic neck X-ray and CT scan that showed complete bony fusion. All patients were then followed for an additional 1.5 years (mean follow-up of 21 months for all patients). No patient showed any neurologic symptoms or deficits during the follow-up period.

Conclusions: In patients with pure C2 body fracture, non-operative management with Philadelphia neck collar is a safe and efficacious option, even in the presence of some sort of ligamentous injury.

Keywords: Axis; Vertebral body; Spinal fractures; Treatment outcome

\section{Introduction}

Axis body fractures, also termed miscellaneous axis fractures and non-odontoid non-hangman's axis fractures, are a relatively less common subgroup of axis (C2) fractures [1-3]. Although C2 fractures compromise $20 \%$ of cervical fractures, C2 body fractures account for approximately $10 \%$ of the upper cervical fractures $[3,4]$.
They are two systems of classification of axis body fractures. The more commonly used one is based on the orientation of fracture line, and classifies these fractures as coronal (type 1), sagittal (type 2), and transverse (type 3) fractures $[1,4]$. The other system classifies them based on imaging findings into avulsion, burst, sagittal, and transverse (or horizontal) fractures (Fig. 1) [5].

While the treatment paradigm for other $\mathrm{C} 2$ fractures

\footnotetext{
Received Dec 4, 2015; Revised Feb 17, 2016; Accepted Feb 26, 2016

Corresponding author: Rouzbeh Motiei-Langroudi

Division of Neurosurgery, Department of Surgery, Pastor Hospital, Bam University of Medical Sciences,

Pastor Hospital, Bam-Zahedan Exp Way, Bam, Iran, 7661771967

Tel: +98-34-443-9401, Fax: +98-34-4251-0847, E-mail: r_motiei@yahoo.com
} 
(odontoid and hangman's fractures) are well described in the literature, there is insufficient evidence to support treatment standards or guidelines for C2 body fractures, as a result of their less common reports [2]. However, in most series, conservative and non-operative management using external immobilization is recommended for treatment of isolated C2 body fractures [1,2,4-7]. Surgical management of this subtype is advocated for a minority of cases and has only been recommended in fractures associated with obvious instability of adjacent joints, irreducible displaced superior articular facet fracture, spinal cord compression [1], and severe malalignment of the atlantoaxial (C1-C2) joint [5].

Here, we present our experience with pure C2 body fractures not accompanied by other cervical spine fractures, and their radiologic and magnetic resonance imaging (MRI) findings, in a group of patients all managed non-operatively by external immobilization. The outcomes of patients including their symptoms and imaging

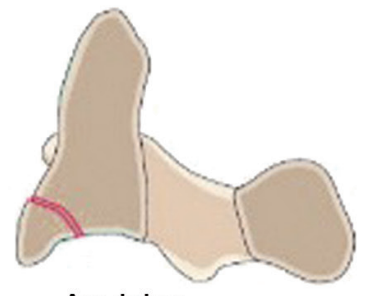

Avulsion

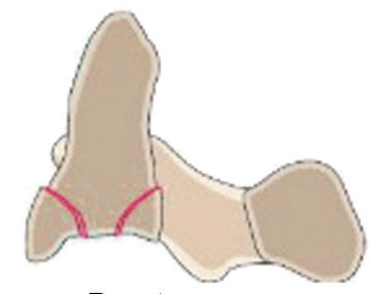

Burst

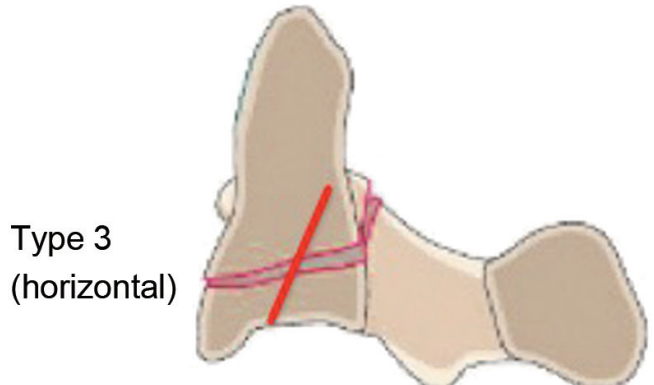

Type 1 (vertical coronal)

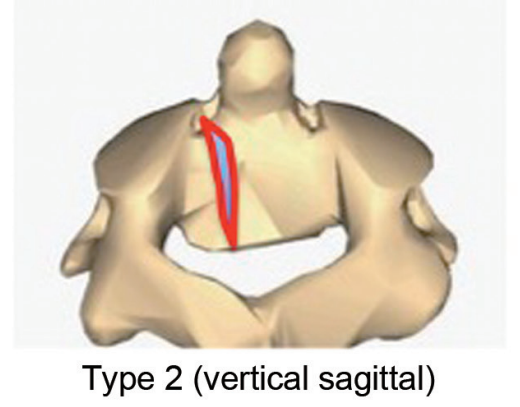

Fig. 1. A schematic illustration of different C2 body fracture types. findings are also presented.

\section{Materials and Methods}

From December 2013 to August 2014, 11 patients with C2 fractures were diagnosed and further evaluated in Pastor Hospital, Bam, Iran. Only patients with pure C2 body fractures were included in this study. Patients with odontoid fracture, hangman's fracture, pars interarticularis or facet fractures were excluded, as were C2 body fractures associated with fractures elsewhere in the cervical spine (e.g., C1).

After primary surveillance in the emergency room (ER) by the trauma management team (including an emergency medicine specialist, neurosurgeon, general surgeon, and orthopedic surgeon), anteroposterior and lateral cervical $\mathrm{X}$-rays, cervical thin $(1 \mathrm{~mm})$ cut computed tomography (CT) scans with sagittal, coronal, and three-dimensional reconstruction (16-slice CT detector, Siemens, Forchheim, Germany), and cervical MRI (1.5 T) was done in all patients.

All patients provided informed and written consent to participate. The study design was approved by the Ethical Committee of Medical University and the study adhered to the statements of the Declaration of Helsinki and regulations of the university's Institutional Review Board.

\section{Results}

The mean age of the six males and five females was 33.2 years (range, 11-53 years). The origin of trauma in all patients was motor vehicle accident (MVA). Upon ER arrival, all patients complained of neck pain, pain upon neck motion, and upper cervical tenderness. All neurologic examinations including motor force, deep tendon reflexes, and sensory were normal in all patients.

CT scans revealed coronal vertical fracture in four patients, sagittal vertical fracture in two patients, and horizontal body fracture in three patients (Fig. 2). In two patients, the fracture had a more complex nature more consistent with a burst fracture. No patient showed evidence of spinal canal compression. MRI revealed hematoma and partial rupture in the anterior longitudinal ligament (ALL) in four patients, posterior ligamentous complex (PLC) injury (interspinous ligament hematoma and tear) in one patient, and normal ligamentous structure in six patients. Disc pathology and thecal sac com- 

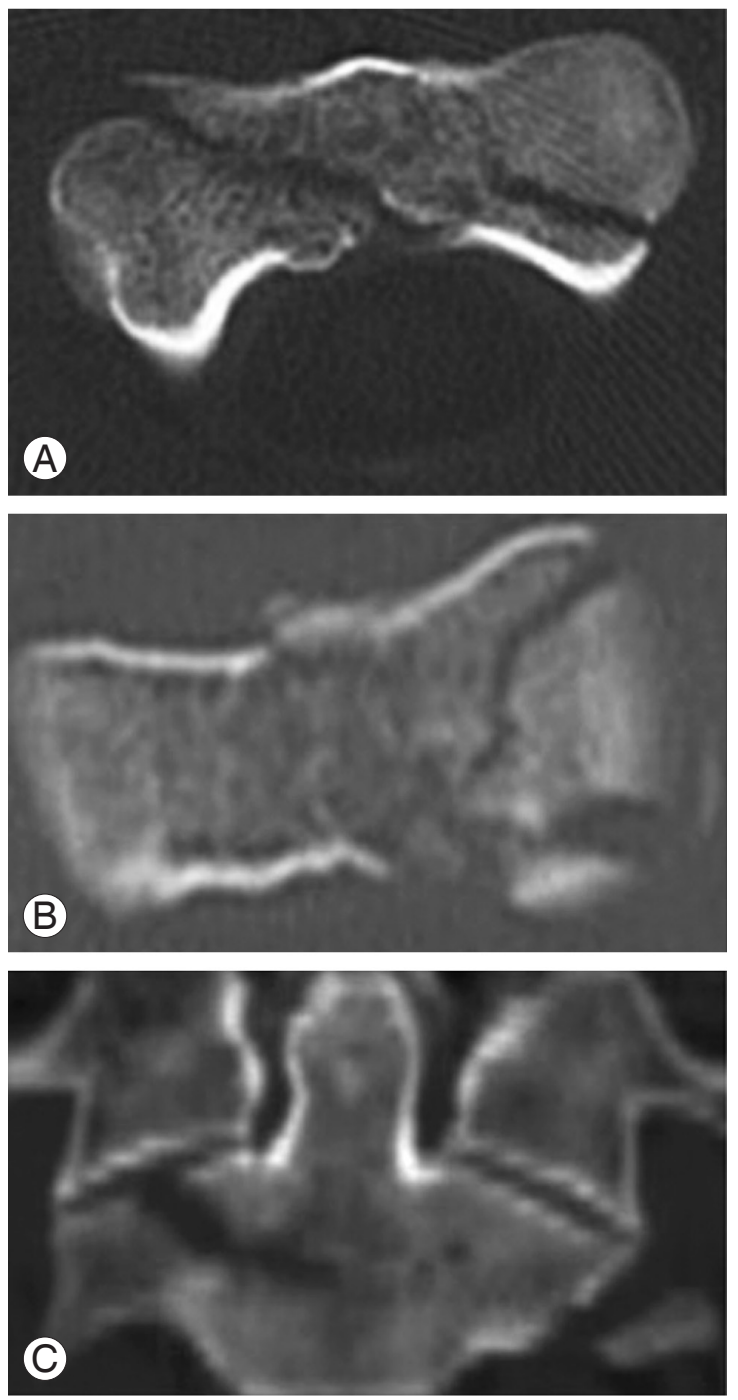

Fig. 2. Computed tomography scan shows a vertical coronal (A), vertical sagittal (B), and horizontal (C) C2 body fracture. pression was not seen in any patient.

The findings are summarized in Table 1. Three patients had clavicle, arm, and leg fractures, which were managed accordingly and separately by an orthopedic surgeon. No damages to internal organs (lungs, intraperitoneal organs, etc.) were detected.

All fractures were managed conservatively by external immobilization gained through a Philadelphia neck collar. Patients had a follow-up visit every month. Collar fixation was continued until symptoms like neck pain had completely disappeared. The time for symptom resolution was 1, 2, and 3 months in two, seven, and two patients, respectively. The decision to discontinue the neck collar was then made by a dynamic neck X-ray and thin cut CT scan from C2 that showed evidence of complete bony fusion and fusion of the associated cervical spinal fractures without instability in all patients (Fig. 3). As evident in $\mathrm{X}$-rays and CT scans, no mal-union or non-union occurred and there were no collar-related complications like skin sores. After discontinuing the neck collar, all patients were followed-up on a regular basis for an additional 1.5 years (mean follow-up in all patients, 21 months; range, 20-22 months). No patient showed any increased neurologic symptoms or neurologic deficits during the followup period. On the last follow-up visit, all patients had regained their previous level of social function, with all adults continuing their previous job and one teenager resuming school without difficulty. According to a $0-10$ visual analogue scale completed by each patient during the last follow-up visit, the median cervical pain was

Table 1. Summary of patients' imaging findings

\begin{tabular}{lccccc} 
Patients' initials & Age $(\mathrm{yr})$ & Sex & Fracture type & MRI finding & Other organs damage \\
AS & 11 & Male & Coronal & NL & No \\
MD & 29 & Male & Burst & PLC injury & Clavicle fracture \\
\hline VR & 28 & Male & Coronal & NL & Tibia fracture \\
FS & 32 & Female & Sagittal & NLL injury & No \\
KB & 35 & Female & Coronal & ALL injury & No \\
JA & 43 & Male & Horizontal & ALL injury & No \\
FJ & 29 & Female & Sagittal & NL & No \\
ZM & 53 & Female & Horizontal & ALL injury & Humerus fracture \\
KZ & 36 & Male & Horizontal & NL & No \\
SB & 44 & Female & Burst & Coronal & No \\
MA & 25 & Male & & \\
\hline
\end{tabular}

MRI, magnetic resonance imaging; NL, normal; PLC, posterior ligamentous complex; ALL, anterior longitudinal ligament. 

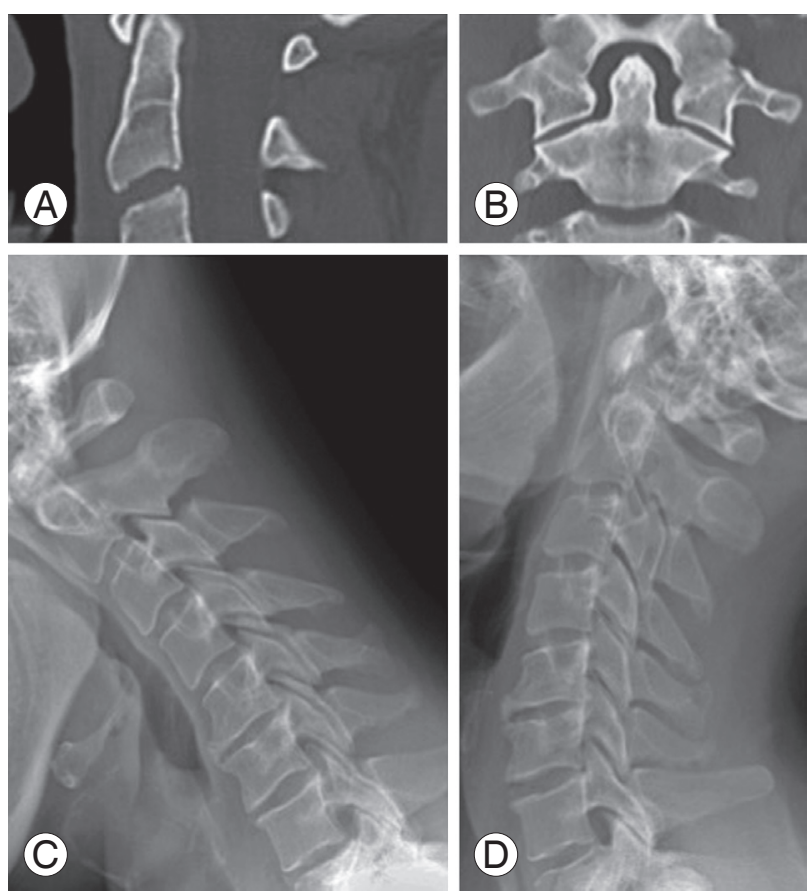

Fig. 3. Follow-up computed tomography (CT) scan (A, B) and dynamic (flexion-extension) $\mathrm{X}$-ray of a patient (C, D). CT revealed complete bony fusion. No mal- or non-union was observed. The $\mathrm{X}$-rays showed no evidence of instability.

2 (range, $0-3$ ). No neurologic or medical complications were reported.

\section{Discussion}

Axis (C2) fractures are categorized based on the anatomical location of fracture line. Axis body fractures, also known as miscellaneous axis fractures or non-odontoid non-hangman's axis fractures, are relatively less common in comparison to odontoid (dens) and hangman's fractures [1-3]. Although C2 fractures compromise $20 \%$ of cervical fractures, $\mathrm{C} 2$ body fractures account for approximately $10 \%$ of the upper cervical fractures $[3,4]$. Because of high levels of motility, and bearing the weight of cranium, the cervical spine is very susceptible to impact injury and fracture. Atlas (C1) and $\mathrm{C} 2$ vertebra, which are totally different in their anatomy compared to subaxial (C3-C7) levels, transmit the weight of the cranium to the more caudal cervical spine, contributing to a higher incidence of fractures [8]. However, different trauma mechanisms may cause different fracture subtypes in this region; C2 body fractures, for instance, may be caused by extension forces [3].
Generally, C2 body fractures are classified based on the orientation of fracture line. Using this classification system yields three types: vertical coronal (type 1), vertical sagittal (type 2), and transverse (type 3) [1,4]. The latter was once regarded as a type 3 odontoid fracture, while most consider it a separate type under $\mathrm{C} 2$ body fracture $[2,3]$. Another lesser used system classifies the fractures based on imaging findings into avulsion, burst, sagittal, and transverse (or horizontal) fractures (Fig. 1) [5].

Given the paucity of cases in the literature, a standard treatment guideline has not been proposed for $\mathrm{C} 2$ body fractures, unlike other $\mathrm{C} 2$ fracture types (odontoid and hangman's fractures) [2]. However, most authors advocate a more conservative non-operative approach in the management of this fracture subtype [1,2,4-7]. In a retrospective review of 18 patients with type 1 and $2 \mathrm{C} 2$ body fractures, all patients were managed non-operatively with external orthoses and immobilization and showed evidence of complete fusion at the time of the last followup, 8 years after injury [4]. Some series, however, have tried to elucidate indications for surgery. A retrospective analysis of 28 patients with C2 body fractures supported non-operative management as the initial therapy [1]. However, the authors advocated surgery in a minority of patients including fractures with unstable adjacent joints, irreducible displaced superior articular facet, and spinal cord compression. The approaches used in their series were posterior $\mathrm{C} 1-\mathrm{C} 2$ pedicle screw fixation and fusion, posterior $\mathrm{C} 1-\mathrm{C} 3$ screw fixation and fusion, and posterior C2 transpedicular lag screws [1]. Others tried to associate treatment paradigm with radiologic features in 31 patients [5]. They showed successful non-operative management in all avulsion and transverse and most sagittal fractures. However, there were cases of burst fractures requiring surgery. The authors recommended non-operative treatment as initial therapy in most cases, while advocating surgery in cases with severe malalignment of the $\mathrm{C} 1-\mathrm{C} 2$ joint [5].

Our study included 11 patients with vertical (types 1 and 2), horizontal (type 3), and burst C2 body fractures without any other forms of fracture, whether in $\mathrm{C} 2$ or other cervical vertebra (pure C2 body fracture). The mechanism of trauma in all our patients was high velocity MVA. In our series, no neurologic deficit was observed either upon presentation or during follow-up. The extremely low incidence of neurologic deficit in C2 body fractures has been seen and confirmed in previous studies [4,7]. Nearly half of our patients had a ligamentous injury evident in 
MRI, either in the form of ALL hematoma, partial rupture, or PLC injury. Patients tolerated a conservative treatment with neck collar well, and all were symptom-free as soon as 1-3 months after trauma, and the benefits remained stable for at least 1.5 years. Our control imaging findings, bony fusion of fracture, confirmed the clinical findings.

\section{Conclusions}

Although our case series compromised a small number of patients, the results could suggest that in patients with pure C2 body fracture, non-operative management with Philadelphia neck collar is a safe and efficacious option, even in the presence of some types of accompanying ligamentous injury. More studies recruiting more patients are needed to establish treatment standards and guidelines.

\section{Conflict of Interest}

No potential conflict of interest relevant to this article was reported.

\section{References}

1. Zhang YS, Zhang JX, Yang QG, Shen CL, Li W, Yin
ZS. Surgical management of the fractures of axis body: indications and surgical strategy. Eur Spine J 2014;23:1633-40.

2. Isolated fractures of the axis in adults. Neurosurgery 2002;50(3 Suppl):S125-39.

3. Benzel EC, Hart BL, Ball PA, Baldwin NG, Orrison WW, Espinosa M. Fractures of the C-2 vertebral body. J Neurosurg 1994;81:206-12.

4. German JW, Hart BL, Benzel EC. Nonoperative management of vertical C2 body fractures. Neurosurgery 2005;56:516-21.

5. Fujimura Y, Nishi Y, Kobayashi K. Classification and treatment of axis body fractures. J Orthop Trauma 1996;10:536-40.

6. Bohay D, Gosselin RA, Contreras DM. The vertical axis fracture: a report on three cases. J Orthop Trauma 1992;6:416-9.

7. Greene KA, Dickman CA, Marciano FF, Drabier JB, Hadley MN, Sonntag VK. Acute axis fractures: analysis of management and outcome in 340 consecutive cases. Spine (Phila Pa 1976) 1997;22:1843-52.

8. Teo EC, Paul JP, Evans JH, Ng HW. Biomechanical study of C2 (Axis) fracture: effect of restraint. Ann Acad Med Singapore 2001;30:582-7. 